\title{
Hybrid Congestion Sharing and Route Repairing Protocol for Bluetooth Networks
}

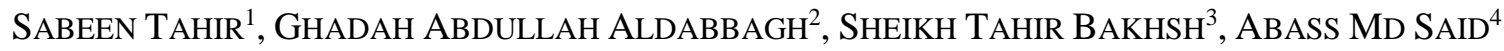 \\ ${ }^{1,2,3}$ Department of Computing and Information Technology \\ King Abdul Aziz University, Jeddah, KINGDOM OF SAUDI ARABIA \\ ${ }^{4}$ Department of Computer and Information Science \\ Universiti Teknologi PETRONAS, Bandar Sri Iskandar, 31750 Tronoh, Perak, MALAYSIA
}

\begin{abstract}
Bluetooth is a widespread wireless technology standard for limited wireless networks that permits Bluetooth devices to create a one-hop (piconet) or multi-hop (scatternet) network. During data transmission, a large number of links passing through a single master or bridge device may create congestion problems in a Bluetooth network. Therefore, routing in a multi-hop dynamic Bluetooth network, where a number of routing masters and routing bridges exist, sometimes create technical problems in a scatternet. Mobility and failure of routing devices disconnects the routing links and link reconstruction process consumes more resources that eventually decrease the performance. In this paper, Hybrid Congestion Sharing and Route Repairing protocol for Bluetooth networks" (HCSRR) is proposed. The objective of this paper is to provide an efficient technique for scatternet congestion avoidance and route maintenance. The proposed protocol is implemented and compared with most relevant protocols. From simulation results, it is observed that the HCSRR outperforms the existing protocols.
\end{abstract}

Keyword-Bluetooth; Hybrid; Congestion; Route maintenance.

Received: January 31, 2021. Revised: April 6, 2021. Accepted: April 16, 2021. Published: April 27, 2021.

\section{Introduction}

Bluetooth technology is a sort of wireless digital and analog communication technology that helps the devices to communicate wirelessly in a short ranged area [1]. As Bluetooth is playing a major role in the communication of handheld electronic device and it is considered as an up and coming standard of Wireless Personal Area Network. Bluetooth uses 79 Radio Frequencies with $2.4 \mathrm{GHz}$ band [8]. In order to avoid the interference it uses the Frequency Hopping Spread Spectrum technique. A hopping sequence can be checked by using the Bluetooth Device Address known as channel. For a given time slot, the frequency of a Bluetooth device can be calculated by the following equation 1 .

$$
f=2402 \mathrm{MHz}+f(t) \times 1 \mathrm{MHz} \text { where } f(t)=0,1,2 \ldots 78 \text {. (1) }
$$

Each channel is divided into time slots of $625 \mu \mathrm{s}$ [2]. Bluetooth devices make a small network up to eight Bluetooth devices known as a piconet or it may create a big ad hoc network known as a scatternet [11]. A scatternet is a combination of multiple piconets where devices from other piconets can communicate by using an intermediate device known as the relay or bridge device. A bridge device can be a slave to slave bridge or master slave bridge. Within a Bluetooth network, devices can play one of many roles like slave, master, bridge or master/slave bridge $[3,12]$. The main duty of a master device is to schedule the data transmission and channel allocation to slave devices. Master transmits its data packets in even numbered slots whereas slave devices transmit their data in odd numbered slots. Firstly, all Bluetooth devices are considered in a standby mode. After initialization Bluetooth devices enters into the inquiry and inquiry scan mode. In order to establish a connection, the master device performs inquiry and slave devices perform inquiry scan operations. In the next step master device performs the page process whereas the slave devices perform page scan procedures. A Bluetooth device can enter into the page scan state from standby or connection state. In order to calculate the average energy consumption, following equation is used.

$$
\begin{aligned}
E_{p s}= & T_{\text {page-scan-window }} E_{r x} \_ \text {idle }+(\text { Tpage-scan - } \\
& T_{\text {page-scan-window) Esb } / T_{\text {page-scan }}} \text { (2) }
\end{aligned}
$$

Where $E_{\mathrm{rx}}$ is used to denote the energy consumption per time slot for scan operation and $E_{s b}$ is used to denote the energy consumption per time slot for sleep mode [15]. After making the communicating links, master device allocates Active Member Addresses AM_Addr to the connected slave devices [13].

In this paper, a hybrid technique is proposed that solves the problem of congestion and route repairing. The intra-piconet congestion is solved by the piconet restructuring. Whereas, the inter-piconet (scatternet) congestion is solved by activating Fall Back Bridge device. On the other hand, the problem of link breakage is solved by the prediction of weak links and weak devices.

The rest of the paper is organized as, Section II discusses the related work and the proposed protocol is presented in Section III. The performance analysis of the proposed protocol and its comparison with a few similar protocols is shown via simulation in Section IV. Finally, the conclusion and possible future work is presented in Section V. 


\section{Related Work}

For intra-piconet

communication, all communicating links go through the master device because direct slave devices cannot communicate with each other. When devices in a piconet communicate very frequently then they may create congestion on a master device. Similarly, direct master devices from other piconet cannot communicate with each other, all the links go through an intermediate bridge device. So, bridge device also have a problem of congestion because of frequently communicating devices from other piconets. Therefore, the master and bridge devices are considered as most important devices. These devices also play an important role in routing. In a scatternet, when data is routed from any source to destination, it follows the route (slave-master-bridge-master-slave) [14]. Any slave from any piconet cannot directly communicate with the other slave of other piconet. During transmission, sometimes link breaks due to the mobility of device or device failure. Many techniques have been implemented for the route maintenance but they only provide the solution when link breakage occurs. Some researchers have been proposed different techniques for congestion avoidance and route maintenance etc. Relay reduction and disjoint route construction protocol [4] were proposed for bridge optimization. A smaller amount of bridges in a scatternet increases the congestion on an active routing bridge device. Due to the congestion on a bridge device, piconets cannot communicate frequently. Therefore, they create delay and prevent parallel transmissions. For piconet congestion sharing, another protocol Dynamic Piconet Restructuring Protocol (PRP) was proposed [5]. The PRP changes the structure of a piconet for congestion sharing. Fig 1 explains the problems in detail.

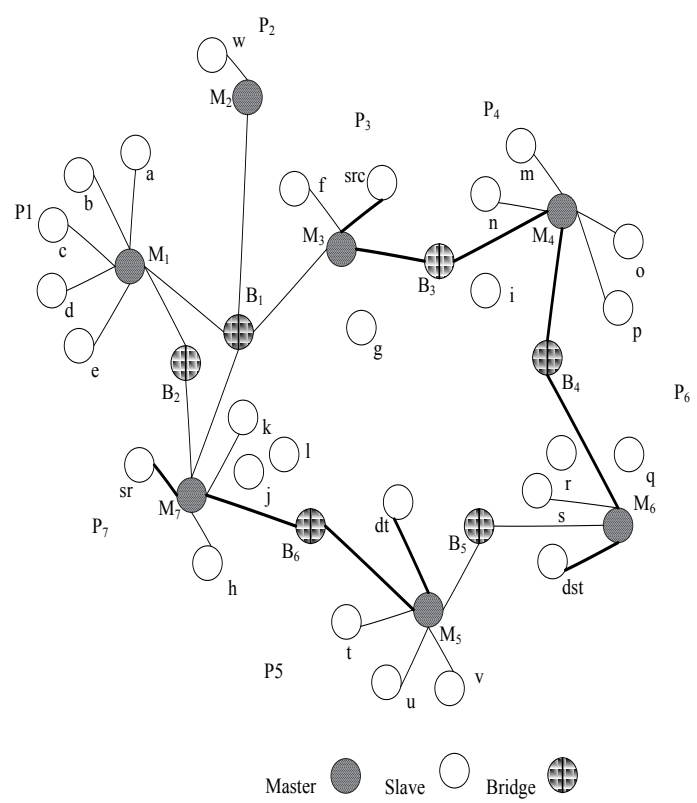

Figure.1: Example of scatternet congestion and route maintenance problems
Suppose piconets, $\mathrm{P}_{1}$ and $\mathrm{P}_{5}$, are considered as congested because the devices inside the piconets are communicating more frequently and ultimately creating the intra-piconet congestion. Piconets, $\mathrm{P}_{1}, \mathrm{P}_{2}$, $\mathrm{P}_{3}$, and $\mathrm{P}_{7}$, are communicating through a single bridge device $\mathrm{B}_{1}$. $\mathrm{B}_{1}$ is used more frequently so it has congestion known as inter-piconet congestion. Devices having congestion decrease the network life time and cannot communicate efficiently.

In the same Fig 1 , two routes $\left(\right.$ src- $-\mathrm{M}_{3}-\mathrm{B}_{3}-\mathrm{M}_{4}-\mathrm{B}_{4}-$ $\mathrm{M}_{6}$-dst) and (sr- $\left.\mathrm{M}_{7}-\mathrm{B}_{6}-\mathrm{M}_{5}-\mathrm{dt}\right)$ have problems of route maintenance. During transmission, the bridge device $\mathrm{B}_{3}$, starts moving and the link between $\mathrm{M}_{3}$ and $\mathrm{M}_{4}$ becomes weak. On the other side, the energy level of $\mathrm{M}_{7}$ starts decreasing and reaches the critical level. In both of these cases, the mobility and minimum energy level of the devices can break the routing link and create major routing drawbacks. Many techniques have been proposed for solving the problem of congestion and route maintenance but they still have to be improved.

The Dynamic Congestion Control (DCC) through the backup relay in the Bluetooth scatternet [6] proposes a solution for scatternet congestion sharing. DCC introduced a new device called the backup relay. A single bridge device is not sufficient for the communication of multiple piconets where devices communicate frequently. It may create congestion. So according to DCC protocol, the master device monitors the traffic load of piconet and delay from bridge device. If there is delay from a bridge device then the master device activates its backup relay device. The DCC protocol works efficiently for sharing the congestion on a master device but it does not show any action for scatternet congestion sharing.

The other relevant protocol is the Novel Route Maintenance (ROMA) [7]. The ROMA protocol works for the Bluetooth ad-hoc network. According to the ROMA protocol, if devices join or leave the network, it decreases the number of hops by reconstructing the routing path. The problem is that, the ROMA protocol does not allow to any routing device which want to communicate with its original master or any other device. The ROMA protocol consumes more time and energy, if any Bluetooth device wants to leave the network because it has to inform the routing master device for node leaving procedure. During transmission, if the routing link breaks or device fails, the ROMA protocol reestablishes the whole network which is more resource consuming. Therefore, it provides an opportunity to propose a new technique of route maintenance that overcome the inefficiencies of existing protocols. 


\section{Proposed Methodology}

In this section, the proposed "Hybrid Congestion Sharing and Route Repairing protocol for Bluetooth networks" HCSRR protocol is discussed.

\subsection{System Model}

The system model including the proposed system and an explanation and the symbols related to the proposed work are as follows:

Auxiliary Master (AxM) and Auxiliary Bridge (AxB):

Any device changes its role and become a master device for the time being is known as an auxiliary master device. Where any device changes its role and become a bridge device is known as an auxiliary bridge device.

Bluetooth Routing devices:

Devices used in a routing link are known as routing devices. These devices can be slave, master or bridge.

Bluetooth Routing Inter-Piconet:

A piconet that contains a routing master device is called as the Bluetooth Routing Inter-Piconet.

Device Information Table (DIT):

According to the HCSRR, within a scatternet, when a new link is established then the routing master and bridge devices maintain their information tables. The routing master device maintains a Routing Master Information Table (RMIT) that contains the list of slave devices, clock offset, device ID, device status, device energy level, signal strength, Fall Back Masters (FBM) and Fall Back Bridges (FBB). Each routing bridge device maintains a Routing Bridge Information Table (RBIT) that contains the list of the connected master devices, device status, and signal strength between two directly connected devices, energy level of a device, FBB list, FBM list and the bridge degree.

Most Frequently used Nodes (MFN):

The devices that communicate again and again for data transmission are known as Most Frequently used Nodes.

Park Mode (PM):

Proposed HCSRR has the ability to change the role of devices when it change the role of any device it also change the mode of a device into Park Mode

Threshold:

Proposed HCSRR uses threshold value to monitor the congestion sharing, energy level and signal strength of devices.

Weak Routing Link and weak device:

A routing link having weak signal strength is considered as a weak routing link. Any device having critical energy level is considered as a weak routing device.
Many scatternet optimization protocols have been implemented but during transmission sometimes they created some serious problems of congestion or route maintenance. These problems ultimately degraded the overall network performance. The main problem with previous protocols is that once the main link broke then they presented the solution for route maintenance that apparently consumed more time, more energy and more control packets. In this paper, the problem of intra-piconet and inter-piconet congestion and the problem of route breakage are solved by using HCSRR protocol. After initializing, it maintains tables for the master and bridge devices. From Fig 2, as the devices in $\mathrm{P}_{1}$ and $\mathrm{P}_{5}$ are communicating more frequently with each other, the proposed HCSRR shares the intra-piconet traffic load by using two techniques Piconet Formation within Piconet (PFP) and Scatternet Formation within Piconet (SFP); as well, it also shares the inter-piconet traffic load on device $\mathrm{B}_{1}$ with the help of the Fall Back Bridge (FBB). The HCSRR technique predicts the weak routing links and the weak routing devices on the basis of mobility and energy level. When it predicts a weak link or weak device it activates the Fall Back Device FBD before the main route breakage.

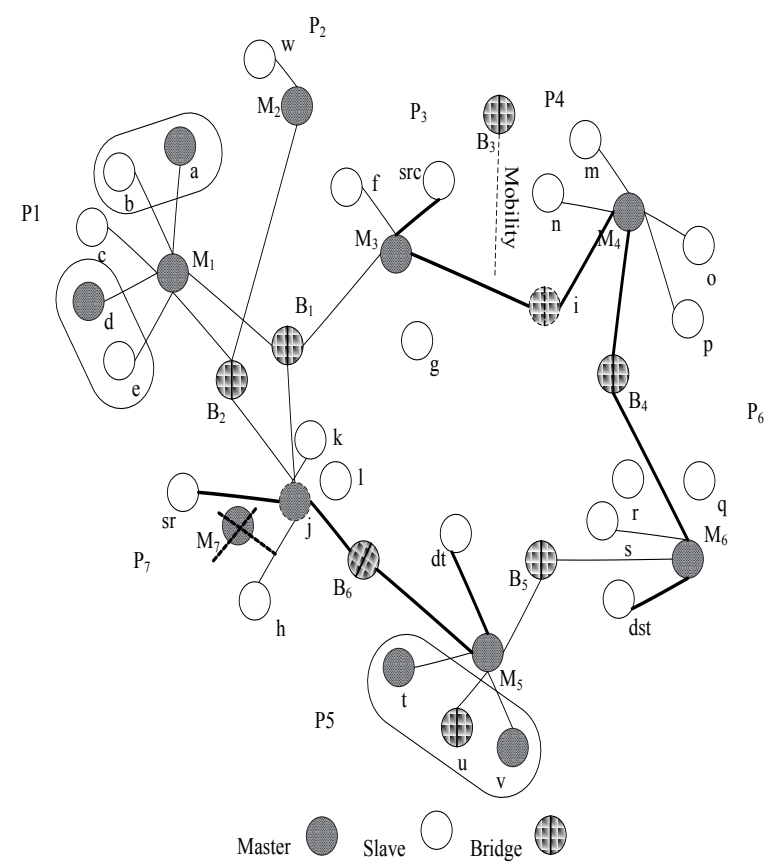

Figure.2: Sharing of congestion and route maintenance by using the HCSRR

The HCSRR performed the role switch operation for the most frequently communicating pairs. For example, $(b, a),(d, e)$ and $(t, v)$ are communicating, repeatedly. The proposed HCSRR used the slave devices (a, d, t, and v) as auxiliary masters and the device $\mathrm{u}$ as the auxiliary bridge to share the congestion on $\mathrm{M}_{1}$ and $\mathrm{M}_{5}$. In piconet $\mathrm{P}_{1}$ it performed the PFP and in piconet $\mathrm{P}_{5}$ it performed the SFP to overcome the congestion problem on the master devices. The bridge device $\mathrm{B}_{2}$, had congestion and it 
did not allow the parallel transmissions between the piconets so the proposed protocol started the transmission by using the $\mathrm{FBB}, \mathrm{B}_{2}$. By activating the FBB, it allowed the parallel transmissions between the piconets and shared the congestion on a single bridge device. In case there was no alternate bridge device, then this protocol could perform the role switch operation to make a new bridge. During transmission, the HCSRR monitored the mobility and energy level of the devices so when it predicted the critical level it activated alternate devices to prevent the route breakage. The HCSRR calculates the signal strength by the following equation.

Where

$$
P \operatorname{Re}=\operatorname{PTr} * G R e^{*} \operatorname{GTr}^{*}(1 /(4 * \pi * d))^{2}
$$

$$
\begin{aligned}
& \text { GTr }=\text { Transmitter antenna gain } \\
& \text { GRe }=\text { Receiver antenna gain } \\
& P T r=\text { Transmit power } \\
& d=\text { distance } \quad\left(\sqrt{(x 2-x 1)^{2}+b(y 2-y 1)^{2}}\right)
\end{aligned}
$$

For example, in the same Fig, during the transmission between source device, src, and destination, dst, the intermediate routing bridge device started moving from its position; when it reached the critical level, the master devices, $M_{3}$ and $\mathrm{M}_{4}$, predicted its mobility and the weak link from their tables and at the same time, they activated the device, $\mathrm{i}$, as a bridge and made a new link between $\mathrm{M}_{3}$ and $\mathrm{M}_{4}$. On the other side, during the transmission between the source, sr, and destination, $\mathrm{dt}$, the energy level of the routing master, $\mathrm{M}_{7}$ in $\mathrm{P}_{7}$, was gradually decreasing which made it as a weak device; when it predicted its decreasing energy level at the same time it activated the FBM device and started the transmission through the FBM device. The main purpose of the HCSRR is to prevent the route breakage and activate a new link before the main route breakage; therefore, it predicts the device energy level and mobility. The hybrid HCSRR technique was implemented and compared with the previous protocols (DCC and ROMA). After getting the simulation results, it was observed that the HCSRR technique outperformed the previous DCC and ROMA protocols.

Concerned Steps of HCSRR technique:

1. Firstly, the master node enters into the inquiry state and the slave nodes enter into the inquiry scan state.

2. Secondly, the master node executes the paging and the slave nodes execute the page scan operations for the synchronisation.

3. In the third step, a constructed and connected scatternet is considered.

4. In the forth step, the master and bridge devices maintain their tables.

5. In the fifth step, the threshold value for the traffic load, energy level and signal strength are defined. As well, the routing links are established.
6. In the sixth step, monitoring of the MF communicating pairs, congestion, energy level and mobility of the devices is performed.

I. If congestion is predicted on a master device, then it performs PFP or SFP:

a) The master node finds the $\mathrm{MF}$ communicating pair of the source and destination.

If they are directly within the radio range of each other then HCSRR performs PFP.

b) The master node sends a request packet for the role switching operation to the pair of the source and destination.

c) The master node updates the record of the source and destination as in the park mode.

d) The source node is selected as an auxiliary master.

e) An efficient PFP is created and the nodes can communicate without creating congestion on a master node.

If the devices are not within the radio range of each other then HCSRR performs SFP.

Then:

a) The master node checks an intermediate node between the source and destination.

b) The master node sends a request packet of the role switching operation to the intermediate node and the pair of the source and destination.

c) The master node selects an intermediate node as $\mathrm{AxB}$ in the same piconet and both the source and the destination nodes are selected as AxMs.

d) The master node updates the record of the $\mathrm{AxB}$ and the pair of the AxMs as in the park mode.

e) Now, an efficient SFP is created and the nodes can communicate frequently.

II. If congestion is predicted on a bridge node, then it activates a FBB.

a) Firstly, the bridge node sends a request packet to the connected masters for the FBB.

b) The master nodes reply with the information of the FBB that can be used to share the congestion.

c) In this step, the master nodes update their status and transmit a request packet of activation to the FBB. Now, the FBB enters into the park 
mode. The FBB makes temporary links between the piconets.

d) Finally, the piconets can communicate through the FBB and efficiently shares the congestion.

After successful communication, the temporary links will be disconnected and the devices will return to their original states for further use.

III. During transmission, if a device having a critical energy level or a device with mobility is predicted, then the HCSRR performs route maintenance. During transmission, the mobility of the devices can be predicted by their weak signal strength. If weak links are predicted because of mobility, then:

a) The routing master device selects a FBD that already exists in its table.

b) The routing master device transmits a request packet to the FBD for the new route activation.

c) The FBD has to make a new link before a main route breakage.

If the FBB is not available, then the HCSRR performs the role switch operation.

d) The master device disables the weak links between the devices and updates the table.

e) Finally, data start going through the new route.

Note: In case a master device starts moving, then, first of all, it will inform all of the connected slave devices and activate its FBM.

IV. If the HCSRR predicts weak links during transmission caused by having a critical energy level, then:

a) It informs the routing master device for the activation of an alternate device.

b) The routing master device selects a FBD from the table.

c) The routing master device transmits a request message of route activation to the FBD.

d) The FBD establishes a new link before the main routing link breakage.

e) The routing master device disables the weak links and updates the table.

f) Finally, data start going through the new route.

Note: If the energy level of the master device starts decreasing, then the master device sends a request message to the FBM device to become a new master device and establishes a new link before the weak link breaks.

\section{Results}

In this sub-section, the comparison results of the HCSRR technique are discussed. The results of this technique have been compared with the results of the previous DCC and ROMA protocols. The DCC and ROMA protocols are selected for the comparison with HCSRR because theses protocols are advanced protocols of congestion sharing and route maintenance. Protocols are implemented in University of Cincinnati's Bluetooth (UCBT) [9] based on the NS-2 simulator [10]. Table 1 shows the list of parameters used during simulation. The Bluetooth devices were scattered in the area of $80 \mathrm{~m} \mathrm{x}$ $80 \mathrm{~m}$, total 90 Bluetooth devices were used, as the proposed protocol works in a dynamic environment so RWMM was used as mobility model. The DH3 and DH5 data packets were used and Round Robin algorithm was set for the scheduling purpose. The CBR traffic model was used for the data flow and the whole simulation execution time was set for $1000 \mathrm{sec}$.

Table1: Simulation parameters

\begin{tabular}{ll}
\hline Parameters & Values \\
\hline Network dimension & $80 \mathrm{~m} \times 80 \mathrm{~m}$ \\
Number of devices & $15-90$ \\
Traffic model & Constant Bit Rate (CBR) \\
Mobility direction & RWMM \\
Scheduling algorithm & Round Robin \\
Number of device pairs & 84 \\
Data packet type & DH3, DH5 \\
Communication range & $10 \mathrm{~m}$ \\
Energy consumption & $0.0763 \times 10^{-6} \mathrm{~J} / \mathrm{bit}$ \\
Inquiry time & $10.24 \mathrm{~s}$ \\
Paging time & $128 \mathrm{~s}-256 \mathrm{~s}$ \\
Simulation time & 1000 \\
\hline
\end{tabular}

\subsection{Total delay}

The total delay of the HCSRR was compared with the delay of the DCC and ROMA protocols. When the congestion arose on the master device, the DCC protocol shared the congestion by activating a backup device. The DCC protocol created more delay because when the congestion occurred on a bridge device, it did not activate backup devices. When one transmission ended, only then did it allow the other transmission; so, during this process, it consumed more time. The ROMA protocol also created more delay because it did not provide the route recovery solution on run time. In this case, when an active link broke, then the ROMA protocol started to find a new link which consumed more time. The HCSRR protocol consumed less time because if it predicted the traffic load on the master device or on the bridge device it performed the role switch operation. The HCSRR performed the role switch operation for more frequently communicating pairs. If it predicted that a single bridge device was providing multiple connections, then it activated the FBB to share the load. During the transmission, the HCSRR recovered the routes when it predicted the weak links and weak 
devices. It activated the FBDs before the main link breakage. The HCSRR technique took less time for the new connection activation which reduced the overall transmission delay. On the other hand, the ROMA protocol did not provide a solution for route recovery on run time. Once an active routing link broke, it started to find a new link which consumed more time as compared to the proposed protocol. From Fig 3, it was analyzed that the total delay of the HCSRR was less than the previous DCC and ROMA protocols.

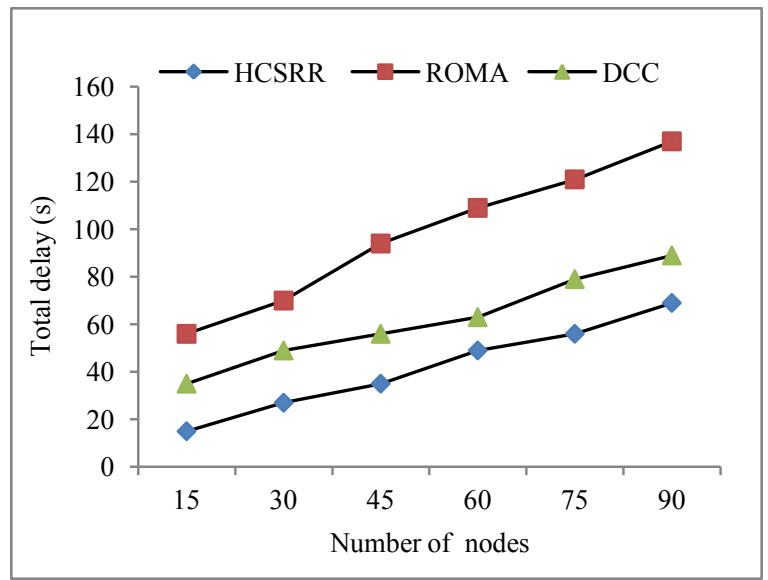

Figure.3: Total delay vs. number of nodes

\subsection{Total control overhead}

The control overhead of the HCSRR was compared with the control overhead of the DCC and ROMA protocols. The previous DCC and ROMA protocols consumed more control packets as compared to the HCSRR. The HCSRR did not perform network restructuring very frequently; when congestion occurred, it only performed network restructuring for the most frequently communicating nodes. From Fig. 4, it was observed that the control overhead of the HCSRR was less than the DCC and ROMA protocols because both of the protocols did not provide a solution for the route maintenance on run time. Whereas, the proposed protocol kept the information of the FBDs, and whenever a weak link or weak device was predicted, it activated the FBD on run time and saved the extra resource utilization. It also prevented the link breakage. Although, the ROMA is a route maintenance protocol, it changed the overall network structure which created unnecessary control overhead. It can be analyzed that the control packet overhead increased in all three protocols with the increase of the received packets.

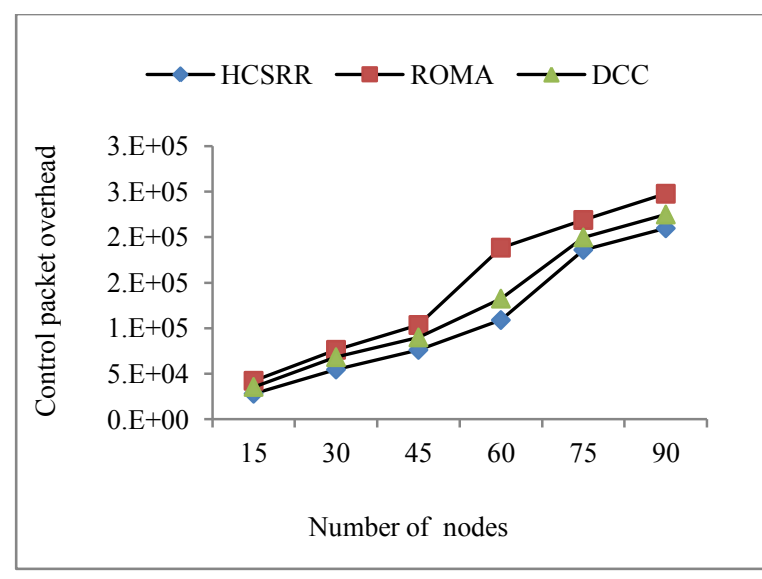

Figure.4: Control packet overhead vs. number of nodes

\subsection{Network Throughput}

It has been observed that the proposed HCSRR increased the network throughput compared to the ROMA and DCC. The reason is that the HCSRR always kept the information of the FBDs for the route maintenance. It repaired the link before the link breakage. By using this way, it prevented the extra resource utilization. On the other side, the ROMA protocol required more time for route maintenance because it maintained the routing links if any device joined or left the network, and for device or link replacement but it did not perform any action if a link suddenly broke or devices failed. It reconstructed the routing path if the communication between the networks stopped; therefore, the ROMA took a higher route recovery time. The DCC only monitored the traffic load on the master node and neglected the bridge node traffic load that increased delay. Fig.5 shows the throughput of the HCSRR, DCC and ROMA protocols. It was predicted from the comparison that the throughput of the HCSRR was higher than the DCC and ROMA.

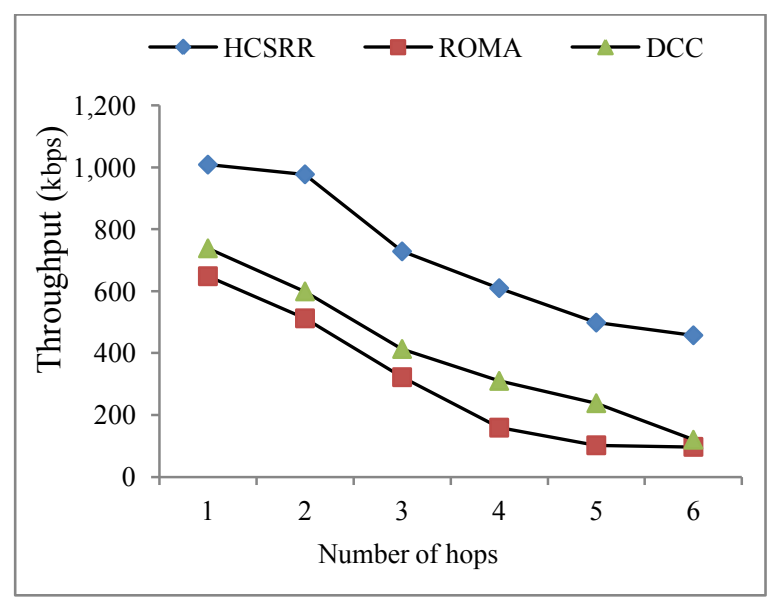

Figure.5: Throughput vs. number of hops

\subsection{Packet loss percentage}

The packet loss percentage of the HCSRR was compared with the packet loss percentage of the DCC and ROMA protocols. In the case of the DCC protocol, the packet loss rate was higher because it 
did not support the congestion avoidance on the bridge node. ROMA is a route maintenance protocol but it did not provide the best solution for the broken links. It maintained a routing link only when a new device joined the piconet or left the piconet. During the transmission, most of the situations occurred when the source and intermediate devices were unable to forward the data to the destination device. Suppose an intermediate link between devices suddenly fails and the data is coming from the source device but an intermediate failed link is unable to forward the data to the destination device. During this situation, more data packets will not reach the destination. The HCSRR technique overcame the problem of data packet loss. It performed route maintenance based on the prediction of the energy level and the mobility of the devices. Therefore, it activated an alternate link as it predicted a weak routing link. By using this method, the proposed HCSRR overcame the problem of data packet loss. From Fig. 6, it is observed that the DCC and ROMA protocols lost more data packets compared to the HCSRR.

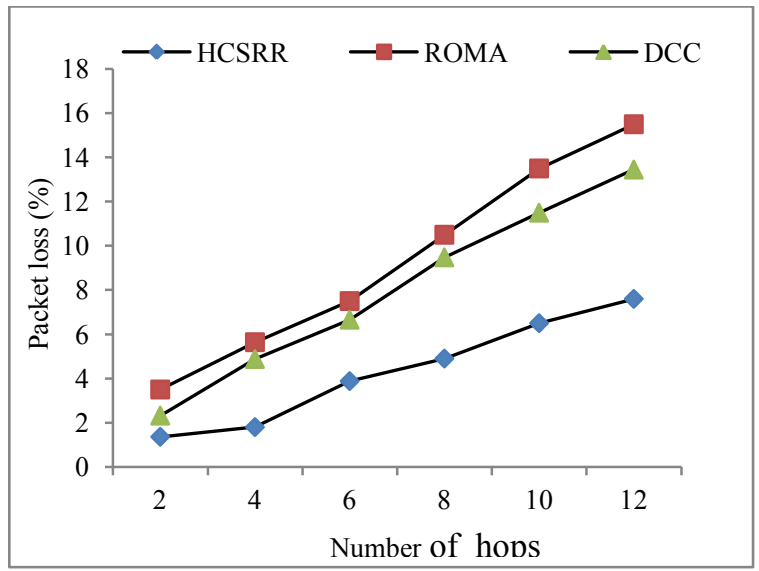

Figure.6: Packet loss vs. number of hops

\section{Conclusion}

In this paper, Hybrid Congestion Sharing and Route Repairing protocol for Bluetooth networks (HCSRR) technique is proposed that shares the Bluetooth network congestion by using the efficient role switching techniques and it also prevent route breakage. The prevention of route breakage based on the prediction of weak links and weak devices. The HCSRR monitors the mobility and energy level of devices when it predicts critical level it activates FBD. This protocol has been implemented and compared with the previous ROMA and DCC protocols. From the comparison results it has been observed that the HCSRR technique performed better in terms of total delay, control overhead, packet loss, route recovery time etc.

\section{References}

[1] Youquan Z, Zhenming F. "Simplifications of the Bluetooth radio devices". In proceedings of IEEE: $4^{\text {th }}$ International Workshop on Networked Appliances.USA, p: $107-115$, 2002.

[2] Bluetooth SIG, Bluetooth Specification v2.0. 2004.

[3] Kallo C.K, Chiasserini C.F, Jung S, Brunato M, Gerla M, "Hop count based optimization of Bluetooth scatternets", Journal of Elsevier: Vol. 5, pp: 340-359, 2007.

[4] Yu G.J, Chang C.Y, Shih K.P, and Lee S.C, "Relay reduction and disjoint routes construction for scatternet over Bluetooth radio system", Elsevier Journal: Network and Computer Applications, Vol. 30(2), pp: 728-749, 2007.

[5] Yu G.J and Chang C.Y, "Congestion control of Bluetooth radio system by piconet restructuring" Elsevier Journal: Network and Computer Applications, Vol. 28, pp: 201-223, 2007.

[6] Bakhsh S.T, Hasbullah H, and Tahir S, "Dynamic Congestion Control through backup relay in Bluetooth scatternet", Elsevier Journal: Network and Computer Applications, Vol. 34(4), pp: 1252-1262, 2011.

[7] Sahoo K.P, Chang C.Y, and Chang S.W, "Novel route maintenance protocols for the Bluetooth ad hoc network with mobility", Elsevier Journal: Network and Computer Applications, Vol. 31(4), pp: 535-558, 2008.

[8] Yu W.C, Yu K.M, and Lin S.L, "Reducing inter-piconet delay for large-scale Bluetooth scatternets", In the Proceedings of Springer: International conference on Emerging technologies in knowledge discovery and data mining, Verlag Berlin, Heidelberg, pp: 562-573, 2007.

[9] University of Cinicinnati Bluetooth simulator (UCBT), Available from: http://www.ececs.uc.edu/ cdmc/ucbt/>, 2010.

[10] The Network Simulator ns-2. Available from: http://www.isi.edu/nsnam/ns/ns-build.html, 2010.

[11] Sharafeddine S, Al-Kassem I, and Dawy Z, "A scatternet formation algorithm for Bluetooth networks with a nonuniform distribution of devices", Elsevier Journal: Network and Computer Applications, Vol. 35(2), pp: 644-656, 2012.

[12] Chakraborty G, "Analysis of the Bluetooth device discovery protocol", Springer Journal: Wireless Networks,Vol. 16(2), pp: 421-436, 2010

[13] Wang Z, Thomas R, and Haas Z, "Performance comparison of Bluetooth scatternet formation protocols for multi-hop networks", Springer Journal, Wireless Networks, Vol. 15(2), pp: 209-226, 2009.

[14] Fang C and Ming H.S, "An Adaptive Interpiconet Scheduling Algorithm Based on HOLD Mode in Bluetooth Scatternets",Journal of IEEE Transactions on Vehicular Technology,Vol. 57(1), pp: 475-489, 2008.

[15] Mei, et al., "Access Control using Bluetooth TM09," Electrical Engineering and Tlececommunications of New South Wales Sydney, Australia, 2003.

\section{Creative Commons Attribution License 4.0 (Attribution 4.0 International, CC BY 4.0)}

This article is published under the terms of the Creative Commons Attribution License 4.0 https://creativecommons.org/licenses/by/4.0/deed.en_US 